Pacific Journal of Mathematics

ANALYTIC FUNCTIONS IN TUBES WHICH ARE
PRESENTABLE BY FOURIER-LAPLACE INTEGRALS 


\title{
ANALYTIC FUNCTIONS IN TUBES WHICH ARE REPRESENTABLE BY FOURIER-LAPLACE INTEGRALS
}

\author{
Richard D. Carmichael and Elmer K. HAYAShI
}

\begin{abstract}
Spaces of analytic functions in tubes in $C^{n}$ which generalize the Hardy $H^{p}$ spaces are defined and studied. In addition Cauchy and Poisson integrals of distributions in $\mathscr{D}_{L}^{\prime}$ are analyzed.
\end{abstract}

1. Introduction. Bochner ([1] and [2]) has defined the Hardy $H^{2}\left(T^{C}\right)$ spaces for tubes $T^{C}=\boldsymbol{R}^{n}+i C$ in $\boldsymbol{C}^{n}$ where $C \subset \boldsymbol{R}^{n}$ is an open convex cone. Stein and Weiss [11] have studied the $H^{p}\left(T^{B}\right)$ spaces for arbitrary $p>0$ and with respect to tubes $T^{B}, B$ being an open proper subset of $\boldsymbol{R}^{n}$ [11, pp. 90-91]. Vladimirov [12, §§ 25.3-25.4] has considered analytic functions in $T^{C}, C$ being an open connected cone, which satisfy the growth [12, p. 224, (64)]. Vladimirov has stated [12, p. 227, lines 4-5] that the growth which defines the $H^{2}$ functions of Bochner is more restrictive than [12, p. 224, (64)]. We show in this paper that the $H^{2}$ growth is not more restrictive than [12, p. 224, (64)] by showing that the functions of Vladimirov are exactly the $H^{2}$ functions. However, Vladimirov's growth has led us to define new spaces of analytic functions in tubes which have growth estimates that are more general than that of the $H^{p}\left(T^{B}\right)$ spaces, and we analyze these new spaces in this paper. Further, we study Cauchy and Poisson integrals of distributions in $\mathscr{D}_{L^{p}}^{\prime}$.

The $n$-dimensional notation in this paper is described in $[7, \mathrm{p}$. 386]. The definitions of a cone in $\boldsymbol{R}^{n}$, projection of a cone $\operatorname{pr}(C)$, compact subcone, and dual cone $C^{*}=\left\{t \in \boldsymbol{R}^{n}:\langle t, y\rangle \geqq 0, y \in C\right\}$ of a cone $C$ are given in [12, p. 218]. Terminology concerning distributions is that of Schwartz [10]. The support of a distribution or function $g$ is denoted $\operatorname{supp}(g)$. Definitions, properties, and relevant topologies of the function spaces $\mathscr{S}, \mathscr{D}_{L^{p}}, \mathscr{B}=\mathscr{D}_{L^{\infty}}$, and $\mathscr{\mathscr { B }}$ and of the distribution spaces $\mathscr{S}^{\prime}$ and $\mathscr{D}_{L^{p}}^{\prime}$ are in [10]. The $L^{1}$ and $\mathscr{S}^{\prime}$ Fourier and inverse Fourier transforms are defined in [7, pp. 387-388] and [10, p. 250], respectively. The limit in the mean Fourier and inverse Fourier transforms of functions in $L^{p}, 1<p \leqq 2$, and $L^{q},(1 / p)+(1 / q)=1$, are in [8] and [3]. $\mathscr{F}[\phi(t) ; x]\left(\mathscr{F}^{-1}[\phi(x) ; t]\right)$ denotes the Fourier (inverse Fourier) transform of a function in the relevant sense. If $V \in \mathscr{S}^{\prime}$ we denote its Fourier (inverse Fourier) transform by $\mathscr{F}[V]=\hat{V}\left(\mathscr{F}^{-1}[V]\right)$. For $\phi \in L^{p}, 1<p \leqq 2$, the Parseval inequality is 


$$
\|\mathscr{F}[\phi(t) ; x]\|_{L^{q}} \leqq\|\phi\|_{L^{p}}, \quad(1 / p)+(1 / q)=1,
$$

with equality if $p=2$, the Parseval equality.

2. The Cauchy and Poisson kernel functions and technical results. Let $C$ be an open connected cone, $C^{*}$ be the dual cone of $C$, and $0(C)$ be the convex envelope (hull) of $C$. The Cauchy kernel function $[6, \mathrm{p} .201]$ is

(2.1) $K(z-t)=\int_{C^{*}} \exp (2 \pi i\langle z-t, \eta\rangle) d \eta, z \in T^{0(C)}=\boldsymbol{R}^{n}+i O(C), t \in \boldsymbol{R}^{n}$.

To avoid the triviality of $K(z-t)=0$ we assume in this section that $\overline{O(C)}$ does not contain an entire straight line $[12$, p. 222, Lemma 1]. In [6, Theorem 1] one of us proved $K(z-t) \in \mathscr{D}_{L^{q}}$ for all $q,(1 / p)+(1 / q)=1,1<p \leqq 2$, as a function of $t \in \boldsymbol{R}^{n}$ for fixed $z \in T^{o(C)}$. But $\mathscr{D}_{L^{q}} \subset \dot{\mathscr{B}} \subset \mathscr{D}_{L^{\infty}}$ for every $q, 1 \leqq q<\infty$, by [10, pp. 199-200]. We thus have

Lemma 2.1. Let $z \in T^{o(C)}$. As a function of $t \in \boldsymbol{R}^{n}$, (2.2) $K(z-t) \in \dot{\mathscr{B}} \cap \mathscr{D}_{L^{q}}$ for all $q,(1 / p)+(1 / q)=1,1 \leqq p \leqq 2$.

For an open connected cone $C$ the Poisson kernel function [6, p. 204] is

$$
Q(z ; t)=\frac{K(z-t) \overline{K(z-t)}}{K(2 i y)}, \quad z=x+i y \in T^{o(C)}, \quad t \in \boldsymbol{R}^{n}
$$

LEMma 2.2. $Q(z ; t) \in \mathscr{\mathscr { B }} \cap \mathscr{D}_{L^{q}}$ for all $q, 1 \leqq q \leqq \infty$, as a function of $t \in \boldsymbol{R}^{n}$ for arbitrary $z \in T^{o(C)}$.

Proof. Let $\alpha$ be any $n$-tuple of nonnegative integers. By the Leibnitz rule

$$
\begin{aligned}
D_{t}^{\alpha}(Q(z ; t))=\frac{1}{K(2 i y)} \sum_{\beta+\gamma=\alpha} \frac{\alpha !}{\beta ! \gamma !} D_{t}^{\beta}(K(z-t)) D_{t}^{\gamma} \overline{(\overline{K(z-t))},} \\
z=x+i y \in T^{o(C)} .
\end{aligned}
$$

By (2.2) $D_{t}^{\beta}(K(z-t))$ and $D_{t}^{r}(\overline{K(z-t)})$ are in $L^{2} \cap L^{\infty}$ as functions of $t \in \boldsymbol{R}^{n}$. Thus $D_{t}^{\alpha}(Q(z ; t)) \in L^{1} \cap L^{\infty} \subseteq L^{q}, 1 \leqq q \leqq \infty$. Hence $Q(z ; t) \in \mathscr{D}_{L^{q}}$ $1 \leqq q \leqq \infty ;$ and $Q(z ; t) \in \dot{\mathscr{B}}$ also since $\mathscr{D}_{L^{q}} \subset \mathscr{\mathscr { B }}, 1 \leqq q<\infty$.

As a function of $x=\operatorname{Re}(z) \in \boldsymbol{R}^{n}$ for $y \in O(C)$ arbitrary we also have

(2.5) $Q(x ; y)=\frac{K(x+i y) \overline{K(x+i y)}}{K(2 i y)} \in \dot{\mathscr{B}} \cap \mathscr{D}_{L^{q}}$ for all $q, 1 \leqq q \leqq \infty$. 
We conclude this section with two important and useful theorems.

THEOREM 2.1. Let $B$ be an open connected subset of $\boldsymbol{R}^{n}$. Let $1 \leqq p<\infty$ and $A \geqq 0$. Let $g(t)$ be a measurable function on $\boldsymbol{R}^{n}$ which satisfies

$$
\int_{R^{n}}|g(t)|^{p} e^{-2 \pi p\langle y, t\rangle} d t \leqq M_{A, g}^{p} e^{2 \pi p A|y|}, \quad y \in B,
$$

where the constant $M_{A, g}$ depends only on $A$ and $g(t)$ and not on $y \in B$. Then

$$
F(z)=\int_{R^{n}} g(t) e^{2 \pi i\langle z, t} d t, z \in T^{B},
$$

is an analytic function of $z \in T^{B}$ and has an analytic extension to $T^{o(B)}$.

Proof. For arbitrary $y_{0} \in B$ there is an open neighborhood of $y_{0}, N\left(y_{0}\right) \subset B$, and a $\delta>0$ such that $\left\{y:\left|y-y_{0}\right|=\delta\right\} \subset N\left(y_{0}\right)$. There are $k$ cones $\Gamma_{j}, j=1, \cdots, k$, having the properties as in [11, p. 92, lines 12-15] and such that whenever two points $v$ and $w$ are in a $\Gamma_{j}$ then $\langle v, w\rangle \geqq(\sqrt{2} / 2)|v||w|$. For each $j=1, \cdots, k$ choose $y_{j}$ such that $\left(y_{0}-y_{j}\right) \in \Gamma_{j}$ and $\left|y_{j}-y_{0}\right|=\delta$. Then for each $p, 1 \leqq p<\infty$, and all $t \in \Gamma_{j}, j=1, \cdots, k$, we have $\left(-2 \pi p\left\langle y_{j}-y_{0}, t\right\rangle\right) \geqq \varepsilon|t|$ where $\varepsilon=\sqrt{2} \pi p \delta>0$. Using this fact, (2.6), and analysis as in [11, pp. 92-93] we have that the function

$$
G(t)=g(t) \exp (\varepsilon|t| / 2 p) \exp \left(-2 \pi\left\langle y_{0}, t\right\rangle\right), t \in \boldsymbol{R}^{n}, 1 \leqq p<\infty,
$$

is an $L^{1}$ function. If $y=\operatorname{Im}(z)$ is restricted so that $\left|y-y_{0}\right|<(\varepsilon \mid$ $4 \pi p$ ) then

$$
\left|g(t) e^{2 \pi i\langle z, t\rangle}\right| \leqq|G(t)|, \quad t \in \boldsymbol{R}^{n}, \quad x=\operatorname{Re}(z) \in \boldsymbol{R}^{n} .
$$

Since $y_{0} \in B$ was arbitrary it follows that $F(z)$ is analytic in $T^{B}$ and has an analytic extension to $T^{O(B)}$ by $[4$, p. 92, Theorem 9].

Note the indicatrix function $u_{C}(t)$ of a cone $C$ defined in $[12, \mathrm{p}$. 219]. $\overline{O(C)}$ may or may not contain an entire straight line in the next theorem.

THEOREM 2.2. Let $C$ be any open connected cone and $A \geqq 0$. Let $g(t) \in L^{p}, 1 \leqq p<\infty$, such that

$$
\int_{R^{n}}|g(t)|^{p} e^{-2 \pi p\langle y, t\rangle} d t \leqq M_{A, \varepsilon, g}^{p} \exp (2 \pi p(A+\varepsilon)|y|), \quad y \in C,
$$

for all $\varepsilon>0$ where the constant $M_{A, \varepsilon, g}$ depends on $A$, $\varepsilon$, and $g(t)$ 
and not on $y \in C$. Then $\operatorname{supp}(g) \cong S_{A}=\left\{t: u_{c}(t) \leqq A\right\}$ almost everywhere (a.e.).

Proof. Assume $g(t) \neq 0$ on a set of positive measure in $S^{A}=$ $\boldsymbol{R}^{n} \backslash S_{A}=\left\{t: u_{c}(t)>A\right\}$, an open set. Then there exists $t_{0} \in S^{A}$ such that $g(t) \neq 0$ on a set of positive measure in any open neighborhood of $t_{0}$. Using $t_{0} \in S^{A}$ and the continuity of the inner product, there is a point $y_{0} \in \operatorname{pr}(C) \subset C$, a fixed number $\sigma>0$, and a fixed open neighborhood $N_{\eta}\left(t_{0}\right)$ of $t_{0}$ such that $\left.\left(-\left\langle y_{0}, t\right\rangle\right)\right\rangle(A+\sigma)>0$ for all $t \in N_{\eta}\left(t_{0}\right)$. Then

$$
-\left\langle\lambda y_{0}, t\right\rangle=-\lambda\left\langle y_{0}, t\right\rangle>\lambda A+\lambda \sigma>0, \quad t \in N_{\eta}\left(t_{0}\right), \quad \lambda>0 .
$$

Since $y_{0} \in \operatorname{pr}(C) \subset C$ and $C$ is a cone then $\lambda y_{0} \in C$ for all $\lambda>0$ and $\left|y_{0}\right|=1$. Using (2.9) and then (2.8) with $y=\lambda y_{0}$ we have for all $\lambda>0$ that

$$
\exp (2 \pi p(\lambda A+\lambda \sigma)) \int_{N_{\eta}\left(t_{0}\right)}|g(t)|^{p} d t \leqq M_{A, \varepsilon, g}^{p} \exp (2 \pi p \lambda(A+\varepsilon))
$$

and hence

$$
\exp (2 \pi p \lambda(\sigma-\varepsilon)) \int_{N_{\eta}\left(t_{0}\right)}|g(t)|^{p} d t \leqq M_{A, \varepsilon, g}^{p}
$$

for all $\varepsilon>0$. By fixing $\varepsilon>0$ such that $\sigma>\varepsilon>0$ and letting $\lambda \rightarrow \infty$ in (2.11) we obtain a contradiction. The conclusion follows by noting that $S_{A}$ is a closed set.

3. The analytic functions. The base $B$ of the tube $T^{B}=$ $\boldsymbol{R}^{n}+i B$ is an open proper subset of $\boldsymbol{R}^{n}$ in this section.

Let $p>0$ and $A \geqq 0 . \quad V_{A}^{p}=V_{A}^{p}\left(T^{B}\right)$ is the space of all functions $f(z)$ which are analytic in $z \in T^{B}$ and which satisfy

$$
\|f(x+i y)\|_{L^{p}}=\left(\int_{\mathbb{R}^{n}}|f(x+i y)|^{p} d x\right)^{1 / p} \leqq M_{A, f} e^{2 \pi A|y|}, \quad y \in B,
$$

where the constant $M_{A, f}$ depends on $A \geqq 0$ and $f$ and does not depend on $y \in B$.

$V^{p}=V^{p}\left(T^{B}\right), p>0$, is the space of all functions $f(z)$ which are analytic in $T^{B}$ and which satisfy

$$
\|f(x+i y)\|_{L^{p}}=\left(\int_{\mathbb{R}^{n}}|f(x+i y)|^{p} d x\right)^{1 / p} \leqq M_{\varepsilon, f} e^{2 \pi \varepsilon|y|}, y \in B,
$$

for every $\varepsilon>0$ where the constant $M_{\varepsilon, f}$ depends on the arbitrary $\varepsilon>0$ and on $f$ and does not depend on $y \in B$.

The spaces defined above have been motivated by the growth $[12$, p. $224,(64)]$ of Vladimirov; we have denoted them as $V_{A}^{p}$ and 
$V^{p}$ accordingly. Notice that $V^{p}=\bigcap_{\varepsilon>0} V_{\varepsilon}^{p}, p>0$; hence $V^{p} \subseteq V_{A}^{p}$, $A>0, p>0$. The Hardy spaces $H^{p}\left(T^{B}\right)=V_{0}^{p}\left(T^{B}\right), p>0$, [11, pp. 90-91] satisfy $H^{p} \cong V^{p}, p>0$; hence $H^{p} \cong V_{A}^{p}, p>0, A \geqq 0$. There are tubes $T^{B}$ and values of $p$ such that $H^{p}, V^{p}$, and $V_{A}^{p}$ contain nonzero functions and such that $V_{A}^{p}$ contains functions which are not in $H^{p}$ or $V^{p}$.

4. Representations of the analytic functions. Analysis as in $\left[11\right.$, p. 99, Lemma 2.12], the $L^{p}$ Fourier transform theory, $1<p \leqq 2$, and a proof similar to that in [11, pp. 100-101] yield

LEMMA 4.1. Let $B$ be an open connected subset of $\boldsymbol{R}^{n}$ and $B^{\prime} \subset B$ such that $\inf \left\{\left|y_{1}-y_{2}\right|: y_{1} \in B^{\prime}, y_{2} \in B\right\} \geqq \delta$ for some $\delta>0$. Let $f(z) \in V_{A}^{p}\left(T^{B}\right), p>0, A \geqq 0$. There exists a constant $K$ which does not depend on $z \in T^{B^{\prime}}$ such that

$$
|f(z)| \leqq K e^{2 \pi A|y|}, \quad z=x+i y \in T^{B^{\prime}} .
$$

If $1<p \leqq 2$, then

$$
e^{2 \pi\langle y, t\rangle} h_{y}(t)=e^{2 \pi\left\langle y^{\prime}, t\right\rangle} h_{y^{\prime}}(t)
$$

for all $y$ and $y^{\prime}$ in $B$ and for almost every $t \in \boldsymbol{R}^{n}$ where

$$
h_{y}(t)=\mathscr{F}^{-1}[f(x+i y) ; t], \quad y \in B,
$$

is the $L^{q},(1 / p)+(1 / q)=1$, inverse Fourier transform of $f(x+i y)$, $y \in B$.

We now represent some $V_{A}^{p}\left(T^{B}\right)$ spaces using Fourier-Laplace integrals.

THEOREM 4.1. Let $B$ be an open connected subset of $\boldsymbol{R}^{n}$. Let $f(z) \in V_{A}^{p}\left(T^{B}\right), 1<p \leqq 2, A \geqq 0$. There exists a measurable function $g(t), t \in \boldsymbol{R}^{n}$, such that

$$
\left(e^{-2 \pi\langle y, t} g(t)\right) \in L^{q}, \quad(1 / p)+(1 / q)=1,
$$

for all $y \in B$,

$$
\int_{R^{n}}|g(t)|^{q} e^{-2 \pi q\langle y, t\rangle} d t \leqq M_{A, f}^{q} e^{2 \pi q A|y|}, \quad y \in B,
$$

where the constant $M_{A, f}$ depends on $A$ and on $f$ but not on $z \in T^{B}$, and

$$
f(z)=\int_{R^{n}} g(t) e^{2 \pi i\langle z, t\rangle} d t, \quad z \in T^{B} .
$$

Proof. Define $h_{y}(t)$ as in (4.3) and put 


$$
g(t)=e^{2 \pi\langle y, t\rangle} h_{y}(t), \quad y \in B .
$$

By (4.2) $g(t)$ is independent of $y \in B$. From (4.3) and (4.7) we have

$$
e^{-2 \pi\langle y, t} g(t)=\mathscr{F}^{-1}[f(x+i y) ; t], \quad y \in B ;
$$

hence (4.4) holds by the Fourier transform theory. Since $f(z) \in$ $V_{A}^{p}\left(T^{B}\right), 1<p \leqq 2$, (1.1) holds for $\mathscr{F}^{-1}[f(x+i y) ; t]$; and by (4.8) and (1.1) we have

$$
\left\|e^{-2 \pi\langle y, t} g(t)\right\|_{L^{q}} \leqq\|f(x+i y)\|_{L^{p}} \leqq M_{A, f} e^{2 \pi A|y|}, \quad y \in B,
$$

from which (4.5) follows. The Fourier transform theory and (4.8) yield

$$
f(z)=\mathscr{F}\left[e^{-2 \pi\langle y, t} g(t) ; x\right], \quad z=x+i y \in T^{B} .
$$

By Theorem 2.1 the integral on the right of (4.6) is analytic in $T^{B}$ and is the $L^{1}$ Fourier transform of $(\exp (-2 \pi\langle y, t\rangle) g(t)) \in L^{1}, y \in B$. (4.6) now follows by the Fourier transform theory and (4.10).

CoROllary 4.1. Let $C$ be an open connected cone. Let $f(z) \in$ $V_{A}^{p}\left(T^{C}\right), 1<p \leqq 2, A \geqq 0$. There exists a function $g(t) \in L^{q},(1 / p)+$ $(1 / q)=1$, with $\operatorname{supp}(g) \leqq\left\{t: u_{c}(t) \leqq A\right\}$ a.e. such that (4.4), (4.5), and (4.6) hold.

Proof. The existence of a measurable function $g(t)$ such that (4.4), (4.5), and (4.6) hold corresponding to $C$ follows from Theorem 4.1. Let $k>0$ be arbitrary. For any $y \in C$

$$
\begin{aligned}
\int_{|t| \leqq k}|g(t)|^{q} d t & \leqq \int_{|t| \leqq k}|g(t)|^{q} e^{-2 \pi q\langle y, t\rangle} e^{2 \pi q|y||t|} d t \\
& \leqq M_{A, f}^{q} \exp (2 \pi q(A+k)|y|)
\end{aligned}
$$

since $g(t)$ satisfies (4.5). Choose $y_{k}=\left(y_{0}\right) /(A+k), y_{0} \in \operatorname{pr}(C)$, the projection of $C$. Then $y_{k} \in C, k>0$, since $C$ is a cone and $A \geqq 0$. By (4.11) with $y=y_{k}$

$$
\int_{|t| \leqq k}|g(t)|^{q} d t \leqq M_{A, f}^{q} \exp \left(2 \pi q(A+k)\left|y_{k}\right|\right)=M_{A, f}^{q} e^{2 \pi q}
$$

since $y_{0} \in \operatorname{pr}(C)$. From Theorem $4.1 g(t)$ is independent of $y \in C$, and the right side of (4.12) is independent of the arbitrary $k>0$. Hence (4.12) proves $g(t) \in L^{q}$. Theorem 2.2 now yields $\operatorname{supp}(g) \leqq\left\{t: u_{C}(t) \leqq A\right\}$ a.e.

The next result follows by the techniques used to prove Theorem 4.1 and Corollary 4.1 together with the facts that $\left\{t: u_{C}(t) \leqq 0\right\}=C^{*}$ and measure $\left(C^{*}\right)=0$ if $\overline{O(C)}$ contains an entire straight line [12, p. 222, Lemma 1]. 
Corollary 4.2. Let $C$ be an open connected cone. Let $f(z) \in$ $V^{p}\left(T^{C}\right), 1<p \leqq 2$. There exists a function $g(t) \in L^{q},(1 / p)+(1 / q)=1$, with $\operatorname{supp}(g) \subseteq C^{*}$ a.e. such that

$$
\int_{\boldsymbol{R}^{n}}|g(t)|^{q} e^{-2 \pi q\langle y, t\rangle} d t \leqq M_{\varepsilon, f}^{q} e^{2 \pi q \varepsilon|y|}, \quad y \in C,
$$

for every $\varepsilon>0$ where the constant $M_{\varepsilon, f}$ depends at most on $\varepsilon$ and $f$; and (4.6) holds for $z \in T^{c}$. Further, if $\overline{O(C)}$ contains an entire straight line then $f(z)=0, z \in T^{c}$.

If we assumed that $g(t) \in L^{q}$ in Corollary 4.2 satisfies $g(t)=$ $\mathscr{F}^{-1}[h(\eta) ; t]$ for some $h \in L^{p}$ then we can prove

$$
f(z)=\int_{R^{n}} g(t) e^{2 \pi \imath\langle z, t\rangle} d t=\int_{R^{n}} h(\eta) K(z-\eta) d \eta, \quad z \in T^{r},
$$

in Corollary 4.2. If $p=2$ the assumption of such a function $h \in L^{2}$ is redundant [3].

Since $H^{p}\left(T^{B}\right) \subseteq V^{p}\left(T^{B}\right), p>0$, and $H^{p}\left(T^{B}\right) \subseteq V_{A}^{p}\left(T^{B}\right), p>0, A \geqq 0$, Theorem 4.1 and Corollaries 4.1 and 4.2 hold for $f(z) \in H^{p}\left(T^{B}\right)$, $1<p \leqq 2$.

Corollary 4.3. Let $C$ be an open connected cone. We have $V^{2}\left(T^{C}\right)=H^{2}\left(T^{C}\right)$.

Proof. Given $f(z) \in V^{2}\left(T^{c}\right)$, Corollary 4.2 yields $g(t) \in L^{2}$ with $\operatorname{supp}(g) \subseteq C^{*}$ a.e. such that (4.13) and (4.6) hold. The Parseval equality (1.1) for $p=2$ yields

$$
\|f(x+i y)\|_{L^{2}}=\left\|g(t) e^{-2 \pi\langle y, t},\right\|_{L^{2}} \leqq\|g\|_{L^{2}} ;
$$

hence $f(z) \in H^{2}\left(T^{C}\right)$. The proof is complete since $H^{p}\left(T^{C}\right) \subseteq V^{p}\left(T^{C}\right)$, $p>0$.

The proof of the preceding corollary combined with the representation $[12$, p. 225, (67)] and the properties obtained for $g(t)$ there show that the analytic functions of Vladimirov in $[12, \S \S 25.3-25.4]$ are exactly the $H^{2}\left(T^{c}\right)=V^{2}\left(T^{c}\right)$ functions.

5. Converse and dual theorems. We now prove a dual result to Theorem 4.1.

THEOREM 5.1. Let $B$ be an open connected subset of $\boldsymbol{R}^{n}$. Let $1<p \leqq 2$ and $A \geqq 0$. Let $g(t)$ be a measurable function on $\boldsymbol{R}^{n}$ which satisfies (2.6). Then the function $F(z), z \in T^{B}$, defined by (2.7) is an element of $V_{A}^{q}\left(T^{B}\right),(1 / p)+(1 / q)=1$. 
Proof. $F(z)$ is analytic in $T^{B}$ by Theorem 2.1, which also implies $(\exp (-2 \pi\langle y, t\rangle) g(t)) \in L^{1}, y \in B$; and by (2.6) this function is in $L^{p}$ also, $y \in B$. Thus (1.1) and (2.6) yield

$$
\|F(x+i y)\|_{L^{q}} \leqq\left\|e^{-2 \pi\langle y, t} g(t)\right\|_{L^{p}} \leqq M_{A, g} e^{2 \pi A|y|}, \quad y \in B,
$$

and $F(z) \in V_{A}^{q}\left(T^{B}\right)$ as desired.

CoROLlaRY 5.1. Let $C$ be an open connected cone. Let $1<p \leqq 2$ and $A \geqq 0$. Let $g(t)$ be a measurable function on $\boldsymbol{R}^{n}$ which satisfies (2.6) for every $y \in C$. Then $g(t) \in L^{p}, \operatorname{supp}(g) \leqq\left\{t: u_{c}(t) \leqq A\right\}$ a.e., and the function $F(z), z \in T^{c}$, defined by (2.7) is an element of $V_{A}^{q}\left(T^{C}\right)$, $(1 / p)+(1 / q)=1$.

Proof. Theorem 5.1, the proof of Corollary 4.1, and Theorem 2.2 yield the results.

If $p=2$, Theorem 5.1 and Corollary 5.1 are converses of Theorem 4.1 and Corollary 4.1, respectively. Similarly the next corollary is a converse of Corollaries 4.2 and 4.3 together with (4.14) for $p=2$.

CoROllary 5.2. Let $C$ be an open connected cone. Let $1<p \leqq 2$. Let $g(t)$ be a measurable function on $\boldsymbol{R}^{n}$ such that (4.13) holds with $q$ replaced by $p$ and $M_{\varepsilon, f}$ replaced by $M_{\varepsilon, g}$. Then $g(t) \in L^{p}$; $\operatorname{supp}(g) \subseteq C^{*}$ a.e.; the function $F(z), z \in T^{c}$, defined by (2.7) is an element of $H^{q}\left(T^{C}\right),(1 / p)+(1 / q)=1$; and there exists a function $h \in L^{q}$ such that $F(x+i y) \rightarrow h(x)$ in $L^{q}$ as $y \rightarrow 0, y \in C$, with this boundary value being obtained independently of how $y \rightarrow 0, y \in C$. Further, if $p=2$ then $F(z)$ has the representation (4.14); and if $\overline{O(C)}$ contains an entire straight line then $F(z)=0, z \in T^{C}$.

Proof. Because of previous analysis the only new idea is the boundary value property. Since $g \in L^{p}$ there exists $h \in L^{q}$ such that $h(x)=\mathscr{F}[g(t) ; x]$ in $L^{q}$. Then $(F(x+i y)-h(x))=\mathscr{F}[(\exp (-2 \pi\langle y, t\rangle)$ $g(t))-g(t) ; x]$ in $L^{q}, y \in C$. Using (1.1) and the Lebesgue dominated convergence theorem the proof is completed.

6. Generalized Cauchy and Poisson integrals. Throughout this section $C$ is an open connected cone such that $\overline{O(C)}$ does not contain an entire straight line.

Let $U \in \mathscr{D}_{L}^{\prime}, 1 \leqq p \leqq 2$. By Lemma 2.1, the generalized Cauchy integral of $U$

$$
C(U ; z)=\langle U, K(z-t)\rangle, z \in T^{o(C)},
$$

is a well defined function of $z \in T^{o(C)}$. 
Using similar proofs we see that [6, Lemma 4] holds for $p=1$, and the convergence in [6, Lemma 5] holds in the topology of $\dot{\mathscr{B}}$. The analysis used to prove [6, Theorems 2,9 , and 10] can be adapted where necessary to show that these results hold also for $p=1$, and we have the following extension of these results.

THEOREM 6.1. Let $U \in \mathscr{D}_{L^{p}}^{\prime}, 1 \leqq p \leqq 2$, and let $C$ be an open connected cone. $C(U ; z)$ is an analyic function of $z \in T^{o(C)}$ which satisfies [6, p. 202,(8)] for $z \in T^{C^{\prime}}, C^{\prime}$ being any compact subcone of $O(C)$. For any $\phi \in \mathscr{S}$ we have

$$
\lim _{\substack{y \rightarrow 0 \\ y \in O(C)}} 0\langle C(U ; x+i y), \phi(x)\rangle=\left\langle\mathscr{F}\left[I_{C^{*}}(\eta) \mathscr{F}^{-1}[U]\right], \phi(x)\right\rangle
$$

with the transforms being in the $\mathscr{S}^{\prime}$ sense. If $U=\hat{V}$ where $V \in \mathscr{S}^{\prime}$ with $\operatorname{supp}(V) \subseteq C^{*}$, then $V=\sum_{|\alpha| \leqq m} t^{\alpha} h_{\alpha}(t), h_{\alpha}(t) \in L^{q},(1 / p)+(1 / q)=1$, for some nonnegative integer $m$; we have

$$
C(U ; z)=\left\langle V, e^{2 \pi i\langle z, t\rangle}\right\rangle, \quad z \in T^{o(C)},
$$

as elements of $\mathscr{S}^{\prime}$; and

$$
\lim _{\substack{y \rightarrow 0 \\ y \in C^{\prime} \subset 0(C)}} 0\langle C(U ; x+i y), \phi(x)\rangle=\langle U, \phi\rangle, \phi \in \mathscr{S} .
$$

[6, Corollary 1, Theorems 11, 12, and 15] hold for $p=1$ also. [6, Theorem 16] can now be extended to include $p=1$ and to conclude the analyticity of $C(U ; z)$ in $T^{o(C)}$, the growth [6, p. 202, (8)] for $z \in T^{C^{\prime}}, C^{\prime} \subset O(C)$, and the convergence (6.2) in each of the connected components $O\left(C_{\lambda}\right), \lambda \in \Lambda$. The restriction of $z \in T^{o(C)} \backslash\{z: y=$ $\operatorname{Im}(z) \in O(C), y_{j}=0$ for any $\left.j=1, \cdots, n\right\}$ in [6, Theorem 16] is unnecessary.

Now let $U \in \mathscr{D}_{L^{p}}^{\prime}, 1 \leqq p \leqq \infty$, and $C$ be an open connected cone. By Lemma 2.2 the generalized Poisson integral of $U$

$$
P(U ; z)=\langle U, Q(z ; t)\rangle, \quad z \in T^{o(C)},
$$

is a well defined function of $z \in T^{o(C)}$. In general $P(U ; z)$ is not analytic. However, if $z$ is in a generalized half plane in $C^{n}$ then $P(U ; z)$ is $n$-harmonic by a proof as in [5, Theorem 7].

We now extend and generalize slightly [6, Lemma 8]. The proof is the same for all $p, 1 \leqq p \leqq \infty$, and for $\phi \in \mathscr{D}_{L^{1}}$ as that indicated for [6, Lemma 8].

Lemma 6.1. Let $U \in \mathscr{D}_{L^{p}}^{\prime}, 1 \leqq p \leqq \infty$, and $z \in T^{o(C)}, C$ being an open connected cone. For $y \in O(C)$ we have 


$$
\langle P(U ; x+i y), \phi(x)\rangle=\langle U,\langle Q(x+i y ; t), \phi(x)\rangle\rangle, \phi \in \mathscr{D}_{L}{ }^{1}
$$

LEMMA 6.2. Let $C$ be an open connected cone and $z=x+i y \epsilon$ $T^{o(C)}$. We have

$$
\lim _{\substack{y \rightarrow 0 \\ y \in O(C)}} \int_{\mathbf{R}^{n}} Q(x+i y ; t) \phi(x) d x=\phi(t), \quad \phi \in \mathscr{D}_{L^{1}}
$$

in the topology of $\mathscr{D}_{L^{q}}$ for all $q, 1 \leqq q \leqq \infty$, and in the topology of $\mathscr{B}$.

Proof. For $y \in O(C)$ and any $n$-tuple $\alpha$ of nonnegative integers (6.8) $D_{t}^{\alpha}(\langle Q(x+i y ; t), \phi(x)\rangle)=\int_{R^{n}} D_{t}^{\alpha}(\phi(x+t)) Q(x ; y) d x, \quad \phi \in \mathscr{D}_{L^{2}}$, where $Q(x ; y)$ is defined in (2.5). $\phi \in \mathscr{D}_{L^{1}}$ implies $\psi_{\alpha}(t)=D_{t}^{\alpha}(\phi(t)) \epsilon$ $\mathscr{D}_{L^{1}} \subseteq \mathscr{D}_{L^{q}}$ for all $q, 1 \leqq q \leqq \infty$. Using [6, Lemma 6, (50)], (6.8), and the analysis of $[6$, p. 214, (55)] and [6, Lemma 7] we have for any $q, 1 \leqq q<\infty$,

$$
\begin{aligned}
\lim _{\substack{y \rightarrow 0 \\
y \in O(C)}}\left\|D_{t}^{\alpha}\left(\int_{R^{n}} Q(x+i y ; t) \phi(x) d x\right)-D_{t}^{\alpha}(\phi(t))\right\|_{L^{q}} \\
\quad=\lim _{\substack{y \rightarrow 0 \\
y \in O(C)}}\left\|\int_{R^{n}}\left(\psi_{\alpha}(x+t)-\psi_{\alpha}(t)\right) Q(x ; y) d x\right\|_{L^{q}}=0
\end{aligned}
$$

which proves (6.7) in the topology of $\mathscr{D}_{L^{q}}$ for all $q, 1 \leqq q<\infty$. Now $\phi \in \mathscr{D}_{L^{1}} \subset \mathscr{\mathscr { B }} \subset \mathscr{D}_{L^{\infty}}$ implies $\psi_{\alpha}(t)=D_{t .}^{\alpha}(\phi(t)) \in \mathscr{D}_{L^{1}} \subset \dot{\mathscr{B}} \subset \mathscr{D}_{L^{\infty}}$. The definition of $\dot{\mathscr{B}}$ implies that $\psi_{\alpha}(t)$ is uniformly continuous and bounded on $\boldsymbol{R}^{n}$; hence the proof of [9, Proposition 3, (b)] yields

$$
\lim _{\substack{y \rightarrow 0 \\ y \in O(C)}} \int_{R^{n}} \psi_{\alpha}(x+t) Q(x ; y) d x=\psi_{\alpha}(t)
$$

uniformly for $t \in \boldsymbol{R}^{n}$. Because of this, (6.9) holds also for $q=\infty$ which proves (6.7) in the topology of $\dot{\mathscr{B}}$ and in the topology of $\mathscr{D}_{L^{\infty}}=\mathscr{B}$.

We now extend and generalize [6, Theorem 14].

THEOREM 6.2. Let $U \in \mathscr{D}_{L^{p}}^{\prime}, 1 \leqq p \leqq \infty$. Let $C$ be an open connected cone and $z=x+i y \in T^{o(C)}$. We have

$$
\lim _{\substack{y \rightarrow 0 \\ y \in O(C)}}\langle P(U ; x+i y), \phi(x)\rangle=\langle U, \phi\rangle, \quad \phi \in \mathscr{D}_{L^{1}}
$$

Proof. The proof follows by (6.6), (6.7), and the continuity of $U$. 
Using Theorem 6.2, [6, Theorem 17] can be extended and generalized for $U \in \mathscr{D}_{L^{p}}^{\prime}, 1 \leqq p \leqq \infty$, where $\overline{O(C)}$ contains no entire straight line. One concludes the existence of $P(U ; z), z \in T^{o(C)}$, and the convergence (6.10) as $y \rightarrow 0, y \in O\left(C_{\lambda}\right), \lambda \in \Lambda$. The restriction of $z \in T^{O(C)} \backslash\left\{z: y=\operatorname{Im}(z) \in O(C), y_{j}=0\right.$ for any $\left.j=1, \cdots, n\right\}$ in $[6$, Theorem 17] is unnecessary.

7. Acknowledgment. One of us, R.D.C., expresses his appreciation to the Department of Mathematics of Iowa State University for the opporunity of serving as Visiting Associate Professor during 1978-1979. The authors thank the referee for his comments and suggestions concerning this paper.

\section{REFERENCES}

1. S. Bochner, Bounded analytic functions in several variables and multiple Laplace integrals, Amer. J. Math., 59 (1937), 732-738.

2. - Group invariance of Cauchy's formula in several variables, Ann. of Math., 45 (1944), 686-707.

3. S. Bochner and K. Chandrasekharan, Fourier transforms, Princeton University Press, Princeton, N. J., 1949.

4. S. Bochner and W. T. Martin, Several complex variables, Princeton University Press, Princeton, N. J., 1948.

5. Richard D. Carmichael, Distributional boundary values in $\mathscr{D}_{L^{p}}^{\prime}$. II, Rend. Sem. Mat. Univ. Padova, 45 (1971), 249-277.

6. - Generalized Cauchy and Poisson integrals and distributional boundary values, SIAM J. Math. Anal., 4 (1973), 198-219.

7. Richard D. Carmichael and E. O. Milton, Distributional boundary values in the dual spaces of spaces of type $\mathscr{S}$, Pacific J. Math., 56 (1975), 385-422.

8. Y. Katznelson, An Introduction to Harmonic Analysis, John Wiley and Sons, Inc., New York, 1968.

9. Adam Korányi, A Poisson integral for homogeneous wedge domains, J. Analyse Math., 14 (1965), 275-284.

10. L. Schwartz, Théorie des distributions, Hermann, Paris, 1966.

11. E. M. Stein and G. Weiss, Introduction to Fourier analysis on Euclidean spaces, Princeton University Press, Princeton, N. J., 1971.

12. V. S. Vladimirov, Methods of theory of the functions of many complex variables,

M. I. T. Press, Cambridge, Mass., 1966.

Received October 19, 1978 and in revised form June 22, 1979.

Iowa State University

AMES, IA 50011

WAKE Forest UNIVERSITY

WINSTON-SALEM, NC 27109

AND

WAKe Forest UnIVERSITY

Winston-SALEM, NC 27109 



\section{PACIFIC JOURNAL OF MATHEMATICS}

\section{EDITORS}

DONALD BABBITT (Managing Editor)

University of Galifornia

Los Angeles, California 90024

HUGO RossI

University of Utah

Salt Lake City, UT 84112

C. C. MOORE AND ANDREW OGG

University of California

Berkeley, CA 94720
J. DUGUNDJI

Department of Mathematics University of Southern California Los Angeles, California 90007

R. FINN AND J. MILGRAM Stanford University

Stanford, California 94305

ASSOCIATE EDITORS

E. F. BECKENBACH

B. H. NeumanN

F. WOLF

K. YosHidA

\section{SUPPORTING INSTITUTIONS}

UNIVERSITY OF BRITISH COLUMBIA UNIVERSITY OF SOUTHERN CALIFONIA CALIFORNIA INSTITUTE OF TECHNOLOGY UNIVERSITY OF CALIFORNIA MONTANA STATE UNIVERSITY STANFORD UNIVERSITY UNIVERSITY OF HAWAII UNIVERSITY OF NEVADA, RENO UNIVERSITY OF TOKYO U'NIVERSITY OF UTAH NEW MEXICO STATE UNIVERSITY WASHINGTON STATE UNIVERSITY OREGON STATE UNIVERSITY UNIVERSITY OF OREGON UNIVERSITY OF WASHINGTON 


\section{Pacific Journal of Mathematics}

\section{Vol. 90, No. $1 \quad$ September, 1980}

Shashi Prabha Arya and M. K. Singal, On the locally countable sum

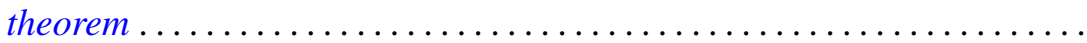

John Theodore Baldwin and David William Kueker, Ramsey quantifiers and

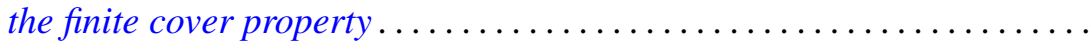

Richard Body and Roy Rene Douglas, Unique factorization of rational

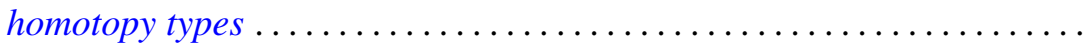

Ethan Bolker and Ben G. Roth, When is a bipartite graph a rigid

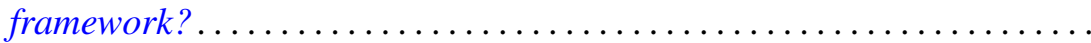

Alicia B. Winslow, Continua in the Stone-Čech remainder of $R^{2} \ldots \ldots \ldots$

Richard D. Carmichael and Elmer Kinji Hayashi, Analytic functions in tubes which are representable by Fourier-Laplace integrals ..............

Stephen D. Cohen, The Galois group of a polynomial with two indeterminate coefficients ..............................

Russell Allan Johnson, Strong liftings commuting with minimal distal

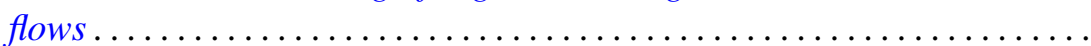

Elgin Harold Johnston, The boundary modulus of continuity of harmonic

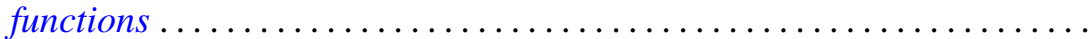

Akio Kawauchi and Takao Matumoto, An estimate of infinite cyclic

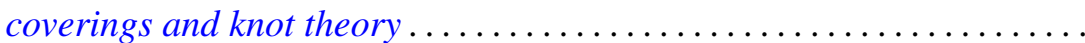

Keith Milo Kendig, Moiré phenomena in algebraic geometry: rational alternations in $\mathbf{R}^{2}$...

Roger T. Lewis and Lynne C. Wright, Comparison and oscillation criteria for selfadjoint vector-matrix differential equations .

Teck Cheong Lim, Asymptotic centers and nonexpansive mappings in conjugate Banach spaces .......................

David John Lutzer and Robert Allen McCoy, Category in function spaces.

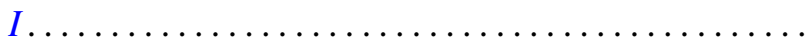

Richard A. Mollin, Induced p-elements in the Schur group ...

Jonathan Simon, Wirtinger approximations and the knot groups of $F^{n}$ in $S^{n+2}$

Robert L. Snider, The zero divisor conjecture for some solvable groups...

H. M. (Hari Mohan) Srivastava, A note on the Konhauser sets of biorthogonal polynomials suggested by the Laguerre polynomials...

Nicholas Th. Varopoulos, A probabilistic proof of the Garnett-Jones theorem on BMO.

Frank Arvey Wattenberg, $[0, \infty]$-valued, translation invariant measures on $N$ and the Dedekind completion of ${ }^{*} R \ldots \ldots \ldots \ldots . .$. 